\title{
Functional Organization of Information Flow in the Corticospinal Pathway
}

\author{
Nofya Zinger, ${ }^{1 \star}$ Ran Harel, ${ }^{2 \star}$ Stephan Gabler, ${ }^{1}$ Zvi Israel, ${ }^{3}$ and Yifat Prut ${ }^{1}$ \\ ${ }^{1}$ Department of Medical Neurobiology, The Hebrew University, Institute for Medical Research Israel-Canada and Edmond and Lily Safra Center for Brain \\ Sciences, Jerusalem 91120, Israel, ${ }^{2}$ Department of Neurosurgery, Sheba Medical Center, Tel Aviv 52621, Israel, and ${ }^{3}$ Department of Neurosurgery, Hadassah \\ Hospital, Jerusalem 91120, Israel
}

Transmission of information in the corticospinal (CS) route constitutes the fundamental infrastructure for voluntary actions. The anatomy of this pathway has been studied extensively, but there is little direct evidence regarding its functional organization. Here we explored the areal specificity of CS connections by studying two related questions: the functional significance of the parallel, motor, and premotor CS pathways; and the way in which finger-related motor commands are handled by this pathway. We addressed these questions by recording from primary motor (M1) and premotor cortical sites in primates (Maccaca fascicularis) performing a motor task, while measuring the evoked intraspinal unit response to single pulse cortical stimulation. Stimulation in M1 evoked spinal neuronal responses more frequently than stimulation in premotor cortex. The number of muscles excited by M1 stimulation was higher than the number excited by premotor stimulation. Within subregions of M1 finger-related sites were sparsely connected with intermediate zone interneurons and tended to affect the ventrally located motoneurons directly. These results suggest that, despite the parallel anatomical organization, the flow of motor commands is predominantly relayed via M1 to downstream elements. The functional impact of premotor cortex is weak, possibly due to inhibitory systems that can shape the flow of information in the CS pathway. Finally, the difference in spinal processing of finger versus wrist-related motor commands points to a different motor control strategy of finger versus wrist movements.

\section{Introduction}

Spinal processing of motor commands is a critical step in translating a motor goal into muscle activation. The primary motor cortex (M1) is the cortical site with the single largest number of corticospinal (CS) fibers (Kuypers, 1973; Murray and Coulter, 1981; Ralston and Ralston, 1985), and its spinal connections were considered to be specifically important for motor control. Nonetheless, anatomical studies have shown that the premotor areas (e.g., supplementary motor area, premotor dorsal, premotor ventral) contain a high density of pyramidal tract neurons, which often was comparable to the density of CS projections found in M1 (Martino and Strick, 1987; Dum and Strick, 1991; He et al., 1993; Dum and Strick, 1996). This finding raised the possibility of a parallel motor pathway through which high-order motor areas gain direct (monosynaptic and excitatory) access to spinal circuitry. To date, studies that used physiological tools have failed to demonstrate this direct connectivity (Shimazu et al., 2004; Boudrias et al., 2010). However, this could reflect the fact that the functional CS connectivity in these studies was estimated indi-

\footnotetext{
Received May 18, 2012; revised Nov. 14, 2012; accepted Nov. 18, 2012.

Author contributions: N.Z. and Y.P. designed research; N.Z., R.H., and Z.I. performed research; N.Z. and S.G. analyzed data; Y.P. wrote the paper.

This work was supported in part by the Israel Science Foundation (ISF-1490/09), The Binational Science Foundation (BSF-2007442), and through the generous support of the Rosetree Foundation and the Baruch Foundation.

${ }^{*} \mathrm{~N}$. Z. and R.H. contributed equally to this work.

Correspondence should be addressed to Yifat Prut, The Hebrew University, Department of Medical Neurobiology, IMRIC and ELSC, Jerusalem 91120, Israel. E-mail: yifatpr@ekmd.huji.ac.il.

DOI:10.1523/JNEUROSCI.2403-12.2013

Copyright $\odot 2013$ the authors $\quad 0270-6474 / 13 / 331190-08 \$ 15.00 / 0$
}

rectly by averaging muscle activity around spike timing (Fetz and Cheney, 1987), a method that is biased toward a strong connectivity with motoneurons and may thus disregard weaker but direct premotor connectivity with spinal neurons.

The aim of this study was to directly estimate the area-based functional organization of CS connectivity by comparing the functional CS connectivity of the premotor versus primary motor cortex. To do so, we simultaneously recorded motor cortical and spinal activity in behaving primates and measured the spinal neural responses evoked by cortical stimulation. We found that functional CS connectivity often deviated from the expected pattern predicted by the anatomical organization of this pathway. First, the density of functional connectivity of premotor sites was significantly lower than the M1 output. Moreover, the unique properties of spinal response to premotor (but not M1) stimulation were consistent with mixed, excitatory-inhibitory premotor control of spinal activity. We further found that, within M1, functional CS connectivity was dependent on somatotopic organization: whereas wrist-related cortical sites were highly connected to the spinal intermediate zone, finger-related sites were mostly directed to ventrally located motoneurons with only little evidence for intermediate-zone collaterals that could affect spinal interneurons. These findings suggest that any parallel motor and premotor impact on spinal activity may be mitigated by inhibitory mechanisms. Hence, despite the parallel anatomical organization, motor-related information is transmitted serially from premotor to motor and from there to spinal circuitry. Finally, the difference between descending systems dedicated to processing finger-related commands and the pathways controlling more 
a cortical
recording/stimulation spinal recording

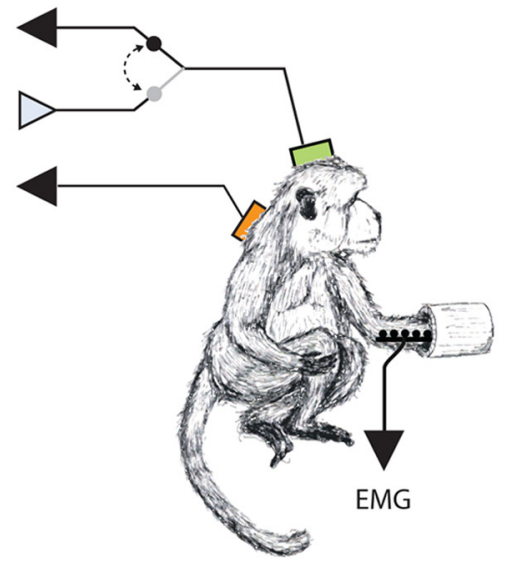

b

step 1: raw signal spinal unit activity

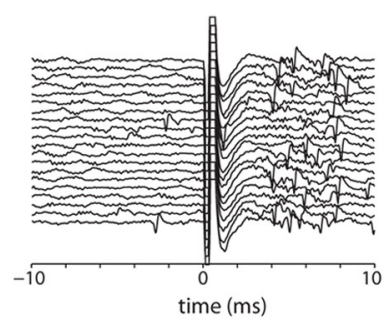

C monkey a

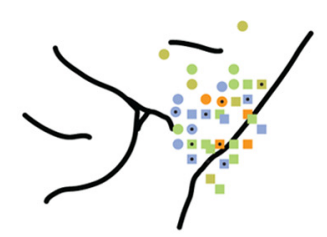

monkey $\mathrm{v}$

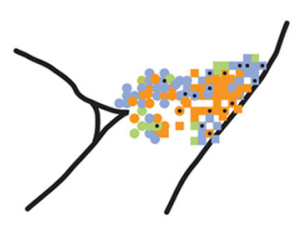

step 2:processed signal highpass-filtered and rectified

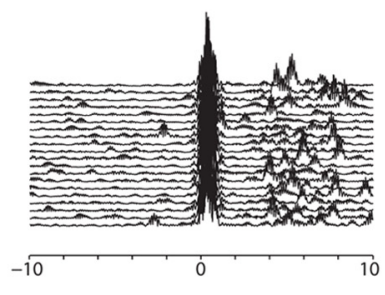

monkey d

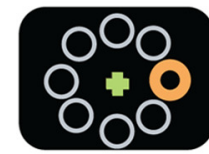

step 3:response quantification

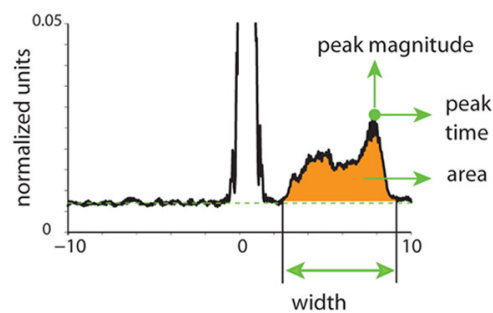

shoulder

-1 elbow

wrist

- fingers

Figure 1. Experimental setting and recording maps. $\boldsymbol{a}$, Monkeys sat in a primate chair and controlled an on-screen cursor by applying an isometric two-dimensional torque at the wrist. Recordings were made from the motor cortex, cervical spinal cord, and arm muscles (EMG). Cortical electrodes were used for both recording and stimulating the tissue. Stimulations (at least 200 single pulses applied at $3 \mathrm{~Hz}$ ) were applied while monkeys performed the task. $\boldsymbol{b}$, To quantify the spinal response to cortical stimulation, the raw spinal recordings (step 1) were processed by high-pass filtering and rectification (step 2). The SD across the sweeps was used to quantify the response. For each significantly responsive spinal site, we calculated the response width, area, peak time, and peak magnitude (step 3).c, Cortical recording maps showing the relations between the recording sites and the nearby sulci. Only arm-related sites that were identified as either M1 (squares) or PM (circles) are shown. Color coding showing the different joint movements evoked by near-threshold stimulation in each site. For monkeys A, D, and V, black dots mark CS connected sites. For monkey C, the black x's show sites where cortical stimulation was applied to measure muscle field size.

proximal joints may indicate a unique control policy for finger movements.

\section{Materials and Methods}

Animals and behavioral task

Four monkeys (Maccaca fascicularis, females, $3-4 \mathrm{~kg}$ ) performed an isometric wrist task with an instructed delay period (Fig. 1a). One monkey (monkey A) performed a one-dimensional flexion-extension task with its hand in mid-pronation position. Three monkeys ( $\mathrm{D}, \mathrm{V}$, and $\mathrm{C}$ ) performed a two-dimensional task with their hand held in either a pronation or supination position. Directional torque at the wrist controlled an on-screen cursor. The details of the behavioral task are described previ- ously (Yanai et al., 2007; Asher et al., 2010) and are not addressed in this study. In short, a trial was composed of a precue period, followed by a visual "cue" indicating the target to attend. After a delay period, a go signal instructed the monkey to shift the cursor by exerting an appropriate directional torque to the previously cued target. Correct performance of the trial (acquisition of the right target within the time limit) was rewarded by a drop of applesauce.

\section{Recording techniques}

A cortical chamber was implanted above the motor cortex, and the location of the arm-related motor cortical areas was mapped using a train of stimulating pulses $(50 \mathrm{~ms}$ of biphasic stimulation delivered at $300 \mathrm{~Hz}$ 
with intensity $\leq 60 \mu \mathrm{A}$ ). Mapping was performed at the end of each recording day with the arm and the hand of the monkey freed from the cast to allow inspection of joint movements in response to the stimulation train. In this study, we selected cortical neurons that were recorded from the arm-related area in the primary motor cortex (threshold for evoked response $<10 \mu \mathrm{A}$ ) or premotor $(\mathrm{PM})$ areas [premotor dorsal (PMd) and ventral (PMv), >15 $\mu \mathrm{A}]$. Note that here we refer to cortical sites in which stimulation evoked responses of the contralateral upper limb (elbow, shoulder wrist, and finger) as arm-related sites. Further identification of recording sites was performed by responses to passive manipulation of the periphery and distance from known anatomical landmarks (e.g., central and arcuate sulci). For monkey C, we found that almost all sites were responsive at a stimulation threshold $<15 \mu \mathrm{A}$. Therefore, for this monkey we used distance from the central sulcus to distinguish between M1 and PM sites. Detailed cortical maps of the four monkeys are shown in Figure $1 c$. Subsequently, a spinal chamber was implanted above the cervical spinal cord (C6-T1). Extracellular singleunit activity was recorded simultaneously from the motor cortex and spinal interneurons located at intermediate lamina while the monkey performed the task (using one to two cortical electrodes and a single spinal electrode). We targeted spinal neurons that were located deep in the intermediate-to-ventral spinal lamina and were recorded deeper than $1 \mathrm{~mm}$ from the first cells encountered. These neurons differed in firing properties from dorsal horn neurons as well as from motoneurons (MNs) (Prut and Perlmutter, 2003a,b). We did not encounter any MN action potentials when averaging muscle activity around spike firing times, although a small proportion of false negatives could have occurred.

Muscle activity (EMG) was recorded using either subcutaneous (monkey V) or transcutaneous (monkey A, D and C) electrodes from selected identified arm and forearm muscles. These included the Flexor-carpi-ulnaris, Palmaris Longus, Flexor-carpi-radialis, Flexordigitorum-superficialis, Flexor-digitorum-profundos, Pronator-Teres, Extensor-carpi-ulnaris, Extensor-digitorum-carpi, Extensor-carpi-radialis, Extensor-digitorum-45, Extensor-digitorum-23, Abductor-pollici-longus, biceps, triceps, and deltoid. After the recordings were completed, two monkeys (A and D) were deeply anesthetized (ketamine, pentobarbital, $30 \mathrm{mg} / \mathrm{kg}$ ), and pins were inserted into known coordinates of the cortical implant. The animals were then killed with pentobarbital sodium $(50 \mathrm{mg} / \mathrm{kg}$, i.v.) and perfused with $10 \%$ formalin or $4 \%$ paraformaldehyde. Cortical locations of penetrations relative to anatomical landmarks were subsequently reconstructed.

\section{Data analysis}

Identifying connected CS sites. To identify spinal responses to cortical stimulation, we measured the evoked multiunit responses (Fig. 1b). Single-pulse stimulations were applied via the recording cortical electrodes at $3 \mathrm{~Hz}$ and various intensities $(50-250 \mu \mathrm{A})$. Stimulation was applied while the monkey performed the task; note that in previous work we found no impact of the specific epoch in which stimulations were applied on response magnitude (Yanai et al., 2007). To test response magnitude, we used at least 200 stimuli.

To identify spinal responses, we first removed the mean stimulation artifact (computed for each stimulation session at the given amplitude) from the single sweep signal. Then, the data were digitally bandpass filtered (eighth-order Butterworth filter, 3-6 kHz) to remove any lowfrequency component that could contain a field effect, and rectified. This processing is equivalent to extraction of a multiunit signal from the compound extracellular signal (Brosch et al., 1997). Averaging the rectified signal is similar in principle to the widely used method of averaging muscle activity around spike timing or applied stimulations (Fetz and Cheney, 1980; Cheney and Fetz, 1985; Bennett and Lemon, 1996; Perlmutter et al., 1998) and reflects a similar purpose: identifying the multiunit response to cortical stimulation. We thus refer to the mean response amplitude in a given time window (either averaged over repeated stimuli or in a single sweep) as a measure of multiunit activity (MUA). To test for significant corticospinal functional connectivity, we used a paired sample Wilcoxon signed rank test to compare multiunit activity in a time window $2-8 \mathrm{~ms}$ after the cortical stimulus onset to a baseline window -10 to $-4 \mathrm{~ms}$ before the cortical stimulus. This was done for a stimulus ampli- tude of $150 \mu \mathrm{A}$, as this stimulation amplitude was common to all tested sites. An example showing the preprocessing of the stimulus-related response is shown in Figure $1 b$.

Quantifying spinal responses. For connected CS sites, we measured the properties of the evoked spinal response by using an inspection window that spanned 1.3-10 ms. The lower bound was set to the earliest expected poststimulus effect (Fetz and Cheney, 1980). We aligned the processed (see above) single sweeps on stimulus onset time and averaged them. We then computed the mean and SD of the averaged trace in the baseline window ( -10 to $-4 \mathrm{~ms}$ before stimulus onset). In the inspection window, we looked for all occurrences of sustained activity $(>0.5 \mathrm{~ms}$, equivalent to 13 samples) above the mean plus 2 SDs of the baseline. Note that for a given average trace, multiple periods of sustained activity can be found. The onset and offset times of such sustained activity were defined to be the first preceding or subsequent bin below the mean plus $1 \mathrm{SD}$, respectively. Response magnitude was the integral of the activity above the mean baseline in all epochs of sustained activity divided by the baseline SD. Onset latency was defined as the start time of the earliest occurrence of sustained activity.

Stimulus-evoked responses of single spinal neurons. We used the compound neural signal (bandpass filtered at $300-6000 \mathrm{~Hz}, \times 10,000$ gain and sampled at 25 or $50 \mathrm{kHz}$ ) and first removed the stimulation artifact by subtracting the average profile of the stimulation artifact. Subsequently, we off-line sorted the compound waveform to extract spikes of single cells (Alpha Sort, Alpha-Omega). We then computed the peristimulus time histogram (PSTH) for single cells in a time frame of -20 to $+20 \mathrm{~ms}$ around stimulation time using a $0.2 \mathrm{~ms}$ bin size. Background firing was computed from -20 to $-10 \mathrm{~ms}$ before stimulus onset. The poststimulation response (starting $2 \mathrm{~ms}$ after stimulus onset) was tested against background firing in a sliding window of $0.4,1$, and $2 \mathrm{~ms}$, shifted in $0.2 \mathrm{~ms}$ steps ( $1 \mathrm{bin}$ ). We $t$ tested the single-sweep firing rates relative to the background firing and identified significant responses, defined as those that deviated from the background level with $p<0.01$ per number of bins (i.e., Bonferroni correction to compensate for the fact that each bin was tested several times). We identified excitatory or inhibitory responses in the time frame of $2-15 \mathrm{~ms}$ defined as a set of at least four successive significant bins. The response of each cell was tested at different stimulation amplitudes since excitation and inhibition could potentially have different threshold levels. We selected cells that were responsive in at least one stimulus intensity. Each of the 27 spinal cells was tested with multiple stimulations intensities, yielding a total of 50 cases in which stimulations were applied in M1, and 17 cases in which stimulations were applied in the PM cortex.

Evoked muscle fields. We computed the evoked EMG response to single-pulse cortical stimulations applied at $3 \mathrm{~Hz}$. To identify the stimulus-induced response for each channel separately, the average activity in a time window ( -20 to $-6 \mathrm{~ms}$ ) before stimulus onset was compared with the average activity in a time window $(6-20 \mathrm{~ms})$ following stimulus onset. The comparison of these trial-by-trial activations was performed across stimulus repetitions using a two-sided rank sum test (Wilcoxon rank sum test). The muscle field size for a session was calculated as the number of channels with significant responses $(p<0.05)$.

Database for studying CS connectivity. We recorded activity from 248 arm-related sites ( 183 in monkey V, 7 in monkey D, and 58 in monkey A) as identified by the evoked movement in response to a brief train of stimuli applied at threshold level. Of these sites, 226 were identified as M1 or PM sites (136 and 90 sites, respectively). PM sites included 74 PMd sites and $16 \mathrm{PMv}$ sites. For this part of the study, we pooled all PM sites.

The remaining 22 sites were either located posterior to the central sulcus, possibly in the somatosensory cortex $(n=3)$, exhibited a mismatch between length (stimulation threshold and anatomical location (high-threshold motor response in M1 sites or low-threshold response in PM sites, $n=15$ ), or we had no XY information regarding the specific penetration site $(n=4)$.

Database for measuring muscle fields. We stimulated in $93 \mathrm{M} 1$ sites (62, 2, and 29 sites from monkeys $\mathrm{V}, \mathrm{D}$, and C, respectively) and $89 \mathrm{PM}$ sites ( 73,1 , and 15 sites from monkeys $\mathrm{V}, \mathrm{D}$, and $\mathrm{C}$, respectively) while recording the activity of forearm muscles. Of these, 93 and 61 sites in M1 and PM, respectively, were identified as arm-related sites. 


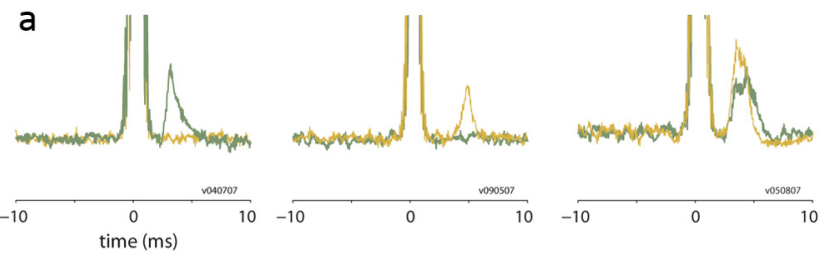

b

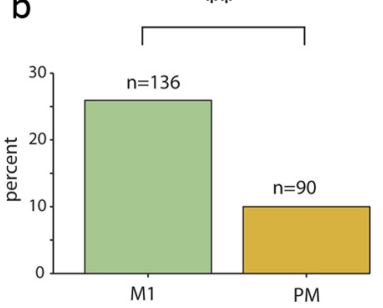

C

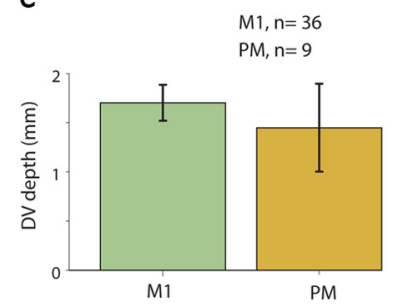

Figure 2. Evoked spinal responses by stimulation in M1 versus PM sites. $\boldsymbol{a}$, Examples of three spinal sites in which responses were evoked by stimulation in M1 (green traces) and/or premotor cortex (brown traces). In all three cases, the M1 and premotor sites were recorded simultaneously in parallel to the spinal recordings. In one case (right-most example), both cortical sites evoked a significant spinal response. $\boldsymbol{b}, \mathrm{M} 1$ sites had a significantly higher tendency to evoke spinal response than PM sites ( $\chi^{2}$ test, $\left.p<0.01\right)$. c, № significant differences were found in the dorsal-to-ventral (DV) depth of spinal sites responding to $\mathrm{M} 1$ and PM stimulation.

\section{Results}

The fraction of directly connected CS sites was higher for M1 sites than for PM sites

Of 248 arm-related cortical sites, we found a total of 49 sites $(21 / 183,4 / 7$, and $24 / 58$ sites in monkeys $\mathrm{V}, \mathrm{D}$, and A, respectively) that evoked significant spinal responses. Of these sites, 45 $(91.8 \%)$ were located in identified M1 or PM sites. Figure $2 a$ presents examples of the mean spinal responses evoked by M1 stimulation (green traces) and PM stimulation (brown traces) in three different spinal sites. Both cortical areas were potentially effective in recruiting spinal neurons even when using singlepulse stimulations. Nonetheless, an area-specific analysis (Fig. $2 b$ ) revealed that the connectivity ratio for $\mathrm{M} 1$ was $\sim 2.5$ times higher than for PM $(26.5 \%$ vs $10 \%$, respectively; a $10.8 \%$ and $6.25 \%$ connectivity rate for PMd and PMv, respectively).

It could be argued that the low probability of PM connectivity was due to a weaker PM-to-spinal connection, which would make it less likely to be detected using our method. In this case, we would have found generally weaker PM-evoked spinal responses compared with spinal responses evoked by M1 stimulations. We quantified the observed spinal responses by their latency, area, and peak value, and found that for all these parameters there were no significant differences that were dependent on the stimulation site (PM vs M1).

An alternative explanation for the low fraction of PM sites that were found to evoke significant spinal responses is that PM sites terminate on a different spinal layer than M1 sites and were thus more often missed in our recordings (since we targeted the intermediate zone and ventrally located interneurons). However, we found that the depth of spinal sites (in the dorsoventral axis) in which M1 evoked significant responses overlapped with the depths of spinal sites responding to PM stimulation (Fig. $2 c$ ). This result confirms that both cortical areas affect the same population of intermediate zone interneurons, consistent with anatomical observations (Dum and Strick, 1996).

\section{The spinal response pattern revealed} direct and indirect activation pathways Cortical stimulation can recruit spinal neurons via both direct and indirect (disynaptic or oligosynaptic) pathways. Previously, we showed that the onset latency of spinal responses to single-pulse cortical stimulations were in the range of $2-4 \mathrm{~ms}$, consistent with monosynaptic latency (Yanai et al., 2007). Nonetheless, neural responses may contain delayed components that are related to the activation of indirect pathways. To estimate the cortical-to-spinal "synaptic distance," we studied the properties of spinal responses after pooling the responses obtained for all stimulation intensities $(n=165$ responses collected for 49 sites; of these, 153 responses were from identified M1 and PM sites). Figure $3 a$ presents typical spinal responses composed of either a single peak (top example) or two successive peaks (bottom example). The
Figure 3. Analysis of response width. $\boldsymbol{a}$, Examples of two typical patterns of spinal responses that present short (single peaks) or long (double peaks) responses. $\boldsymbol{b}$, Distribution of response width computed for M1 (green bars) and PM (brown bars, plotted as a mirror reflection) based on significant spinal responses obtained at all stimulation amplitudes in M1 or PM sites $(n=153)$. A small but significant difference was found between M1 and PM responses ( $t$ test, $p<0.05$ ) that reflects the tendency for PM-evoked responses to be shorter than M1-evoked responses. $c$, Average short (black line) and long (gray line) responses defined using a $4.5 \mathrm{~ms}$ criterion. Stimulation artifacts were first removed, and the single responses were then normalized to emphasize the response shape. Shaded areas around each average reflect the SEM. $\boldsymbol{d}$, the dependency of response width on stimulation amplitude computed for M1 sites (green circles) and PM (brown triangles) sites. Correlation values $(\rho)$ and their significance level are shown as well.
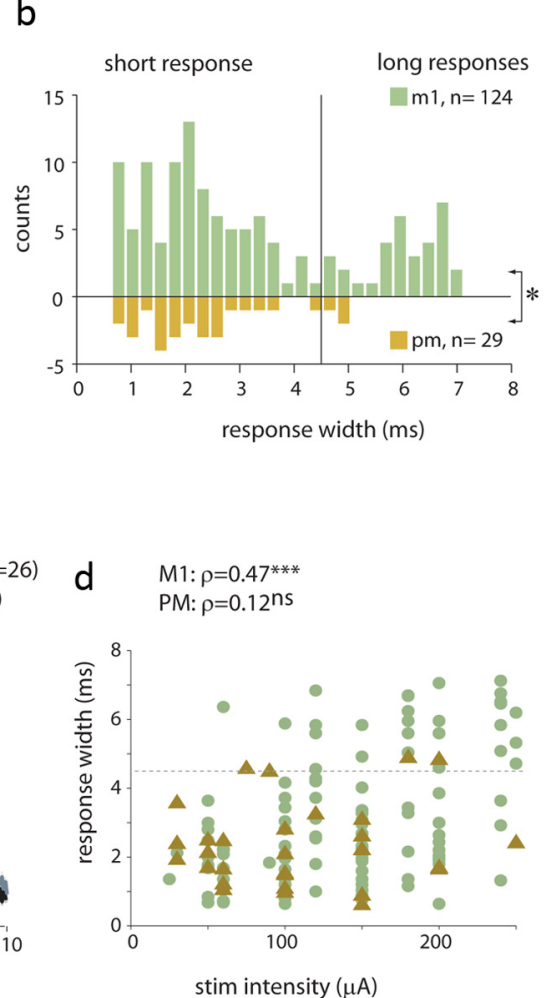
propensity for multiple response peaks was captured by the width of evoked spinal responses (Fig. 3b), which appeared bimodal: narrower effects were usually shorter than $4.5 \mathrm{~ms}$, whereas broader effects were longer than this value. Averaging long and short responses separately (Fig. $3 c$ ) revealed that short and long responses had a similar onset profile and that the main contribution for the extended width was related to an additional response peak. This indicates that the bimodal property of response width reflected the fact that spinal responses were composed of a sequence of one or more essentially discrete events with only a few responses that were single broad peaks.

We further found that the width of PM-evoked responses differed from M1evoked responses in two main aspects. First, spinal responses evoked from M1 were significantly wider than those evoked from PM (Fig. $3 c, d$; $t$ test, $p<$ 0.05). Second, even though the width of M1-evoked responses was strongly correlated with the stimulation amplitude (Fig. $3 d$ ), the width of PM-evoked responses was independent of stimulation magnitude. The lack of a width-intensity correlation was not due to the smaller sample size available for PM responses. The correlation between M1-evoked responses and stimulus amplitude remained significant even when testing the correlation for randomly selected M1 subsets, each containing the same number of sites available for PM data $(n=29)$, or when drawing these subsets while matching the intensity distribution available for PM sites $(p \ll 0.001)$.

\section{Response patterns of single spinal neurons support a PM- derived inhibitory drive}

Studying CS connectivity based on the evoked MUA response provides an unbiased estimate for the interactions, similar to the use of EMG data for studying neuromuscular connectivity (Fetz and Cheney, 1980; Perlmutter et al., 1998). However, the properties of this signal (a strictly positive signal that is specifically sensitive to synchronized changes in firing rate) make it less suitable for studying inhibitory effects. We therefore explored the response pattern of single spinal neurons recorded from sites that were significantly affected by cortical stimulation. Figure $4 a$ presents an example of a single cell that responded to the cortical stimulation pulse (Fig. 4a). Among the responsive spinal cells, we found three typical response patterns (Fig. 4b), which included excitation, inhibition, and an excitation followed by inhibition. We further found that while M1 stimulation tended to induce a pure excitatory response, PM stimulation more frequently induced either inhibitory or excitatory-inhibitory responses. An example of this propensity is shown for a single spinal neuron that was affected by both M1 and PM stimulation; for this neuron, stimulations in M1 induced a pure excitation of the unit, whereas stimulation in PM induced an excitation followed by inhibition (Fig. 4c). For the entire set of responsive neurons, we found that the distribution of response patterns evoked by M1 was statistically significant compared with the patterns evoked by PM stimulation (Fig. $4 d ; \chi^{2}$ test, $p<0.002$ for all cells and $p<$ 0.004 when considering only responsive cells).

\section{The number of muscles affected by cortical stimulation was dependent on the cortical area}

The above results quantified motor and premotor connectivity with spinal interneurons. We further compared these results to the functional connectivity between motor cortex and arm muscles. Cortical connectivity with muscles was quantified by the number of significantly responsive muscles to single-pulse cortical stimulation (i.e., muscle-field size). Significant muscle responses were measured by stimulus-triggered averaging of muscle activity around stimulation time, which can detect subliminal muscle responses that do not induce overt movement of the relevant joints. We found that the average size of muscle fields evoked by M1 stimulation was significantly larger than the size of the muscle field evoked by PM stimulation ( $p \ll 0.001$ Fig. $5 a$ ). This result was obtained when including only finger- and wristrelated sites in M1 and PM (Fig. 5a) as well as when including all tested sites. When testing the dependency of the muscle field size on stimulation amplitude (Fig. 5b), we found that M1 sites were consistently more effective in recruiting muscles than PM sites. Moreover, increasing the stimulation amplitude beyond $150 \mu \mathrm{A}$ (the value that was used for the analyses above) only moderately increased the muscle field size. It thus seems reasonable to assume 


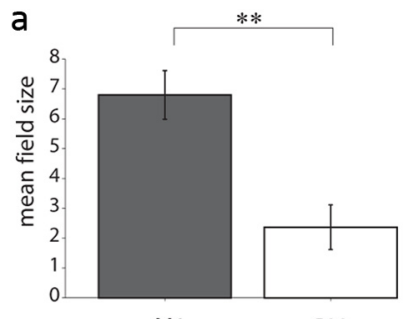

M1

PM

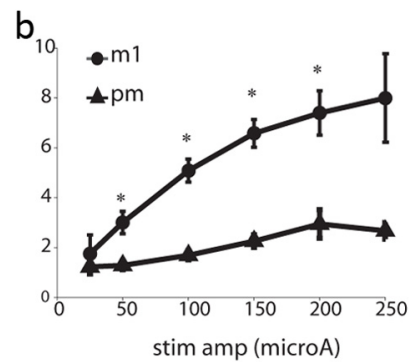

Figure 5. Site-dependency of evoked muscle field. $\boldsymbol{a}$, The muscle field size (i.e., the number of muscles significantly recruited by cortical stimulation) was significantly larger ( $t$ test, $p \ll$ 0.01) for single-pulse stimulations in M1 (dark bar) than for stimulations in PM (light bar). $\boldsymbol{b}$, The intensity dependence of the field size for M1 (circles) and PM (triangles) stimulation as a function of stimulation amplitude. Error bars are provided for each stimulation value, and asterisks denote significant differences in the mean field size between M1 and PM sites.
CS pathways related to different parts of the arm could follow unique organization principles and thus present a different control strategy for different types of movements. We tested this hypothesis by measuring the impact of motor cortical organization on the evoked spinal response. Figure 6 illustrates examples of spinal responses evoked by a finger-related cortical site (Fig. $6 a$ ) and a wrist-related site (Fig. 6b). In M1, we found (as expected) an extensive representation of fingers, yet the tendency of these sites to be connected with spinal interneurons was considerably lower (Fig. $6 c$ ) compared with wrist-related sites. Furthermore, spinal sites that responded to stimulation in finger-related M1 and PM sites were located significantly more ventrally than spinal sites responding to wrist-related sites (Fig. $6 d$ ). By contrast, the segmental levels (on the rostro-caudal axis) of the observed wrist-related sites and finger-related sites overlapped ( $\chi^{2}$ test, $p>0.31$ ). This indicates that finger-related cortical commands are relayed directly to ventrally located motoneurons and are less likely to be processed by the rich interneuronal circuitry.

Interestingly, we found no differences between finger- and arm-related cortical sites in terms of the size of the evoked muscle field (two-way ANOVA, $p_{\text {motor-area }} \ll$ $0.001, p_{\text {arm-site }}>0.33, p_{\text {interaction }}>0.13$ ). This result strengthens our claim that finger-related cortical commands are relayed directly to motoneurons. It further suggests that this relay is not muscle specific, in that the muscle fields of finger-related and arm-related sites were comparable.

\section{Discussion}

The functional organization of the CS pathway has primarily been studied indirectly by measuring functional corticomuscular links using either spike-triggered averages (Cheney and Fetz, 1980) or stimulus- d

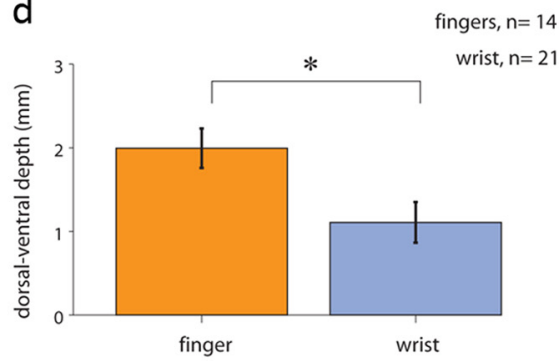
triggered averaging (Cheney and Fetz, 1985). This makes it impossible to differentiate between cortical control of spinal interneurons situated in the intermediate zone and direct cortical control of motoneurons located in the ventral horn. Previously, we showed that functional CS interactions are extensive and dynamic (Yanai et al., 2007; Asher et al., 2010) and play a critical role in the transformation of motor commands into muscle activation signals (Yanai et al., 2008; Shalit et al., 2012). Here we extend these finding by measuring the organization of functional CS connectivity in relation to cortical areas (M1 vs PM) and its detailed joint-related organization (finger vs nonfinger sites).

We found that, despite the highthat the difference in muscle field size evoked by M1 and PM was not a byproduct of some threshold effect that could be compensated for by increasing the stimulation amplitude.

\section{The functional CS connectivity of M1 sites was joint specific} We tested the impact of the somatotopic organization within M1 on the functional connectivity with spinal circuitry. Potentially, density anatomical CS connections previously reported for premotor areas with the spinal intermediate zone (He et al., 1993; Dum and Strick, 1996), only a small fraction of premotor sites was functionally connected with spinal interneurons. We further showed that this result is not an outcome of some technical limitation of our recording technique (e.g., subliminal PM-to-spinal 
connectivity strength); rather, it reflects an inherently sparse pattern of functional connectivity.

This finding was reinforced by the smaller muscle field size evoked by stimulation in PM sites. This result may appear at odds with the concept of motor hierarchy, which assumes that higher motor cortical areas control broader motor concepts (or complex movements) and may thus affect larger groups of muscles (Porter and Lemon, 1993). Our results suggest that the premotor cortex is organized according to a more abstract motor framework that is independent of a specific muscle activation pattern.

When pooling spinal responses of all intensities, we found a significant difference in the width of M1-evoked and PM-evoked responses. Specifically, M1 stimulation applied at high intensities tended to evoke prolonged spinal responses composed of at least two isolated peaks. While the first peak can be attributed to direct CS activation, the later peak reflects a secondary activation pulse converging onto the same spinal target as the direct activation. At this stage, we can only speculate as to the source of the indirect activation, since it could reflect local dynamics (cortical or spinal) or indirect activation via subcortical stations such as the red nucleus (Kuypers and Lawrence, 1967; Monakow et al., 1979; Humphrey et al., 1984). Nonetheless, the timing of these delayed peaks, their magnitude, and termination pattern (which matches the direct pathway) make it more plausible that these peaks are mediated by subcortical structures and not by local cortical and/or spinal mechanisms. In this context, it is puzzling that the premotor cortex, which is connected to spinal interneurons via similar pathways as M1, hardly ever evoked bimodal responses, even when stimulated at high intensities. A possible explanation is that the indirect $\mathrm{PM}$-to-spinal pathway contains both excitatory and inhibitory components, which cancel out at the spinal level.

This hypothesis was further supported by the response patterns of single spinal units evoked by cortical stimulation. Here again, M1 stimulation tended to induce pure excitation, while PM stimulation more often induced inhibitory responses (either exclusively or following an excitatory phase). Note, however, that it is difficult to deduce disynaptic inhibition using extracellular recordings due to the confounding impact of the refractory period, which may appear as an inhibition. Also, disynaptic connections could be masked, at least in part, by the earlier (strongly locked) monosynaptic response. Thus, to confirm or reject our hypothesis more direct measures of PM impact on spinal neurons are needed.

Earlier studies hinted at the putative role of the premotor cortex in inhibiting low-level motor output based on anatomical (Moll and Kuypers, 1977) and physiological evidence (Prut and Fetz, 1999; Duque et al., 2012). Accordingly, the increased premotor activity during movement preparation (Wise et al., 1986; Churchland et al., 2010) can act as a gating signal at the spinal level to prevent premature movements (Prut and Fetz, 1999). Here we provide supporting evidence for a presumably indirect PM-to-spinal inhibition that could counteract direct CS excitation. Clearly, this explanation requires a more direct demonstration, yet it may reconcile the discrepancy between the anatomical findings showing rich CS pathways emerging from premotor areas and our functional evidence suggesting sparse premotor-tospinal connectivity.

In primates, fingers are extensively represented across the primary motor cortex (Schieber, 2002). In parallel, the increased dexterity of primates was shown to be correlated with the evolvement of the direct corticomotoneuronal pathway (Bortoff and Strick, 1993). These results may suggest that finger movements are predominantly controlled by cortical circuitry. However, single tracing analyses of pyramidal tract axons have shown that single fibers that terminate on motoneuron pools tend to send collaterals to the intermediate zone where spinal interneurons are located (Shinoda et al., 1981). More recently, it was shown that spinal neurons are active during finger movements (Takei and Seki, 2010) and that propriospinal systems can help in restoring finger function when the direct CS pathway is damaged (Nishimura et al., 2007) via enhanced premotor control. Our results suggest that finger-related cortical commands are processed very little by spinal interneurons. Instead, finger-related information is transmitted to more ventral sites where motoneurons are situated, to activate a rather nonspecific set of upper limb muscles. This indicates that spinal activation during finger movement is probably induced either by a feedback response or as a result of the corecruitment of additional (non-finger related) cortical sites needed for stabilizing more proximal joints during finger tasks. Another implication of this finding is that the control of arm movements cannot be directly inferred from studies of finger-related motor tasks (Valero-Cuevas et al., 2009) because the difference in use of spinal circuitry in varying motor tasks may imply different control algorithms.

Finally, our results have two main implications. First, the flow of cortical motor commands along the motor hierarchy is predominantly serial; namely, from premotor to primary motor and from there to spinal circuitry. Hence, it is unlikely that the parallel organization of CS pathways is used to independently excite lowlevel motor elements; rather the PM-to-spinal connectivity may act to downregulate segmental excitability. Second, fingerrelated motor commands are processed in a unique pattern compared with other arm-related commands consistent with the late evolutionary emergence of the direct corticomotoneuronal pathway (Heffner and Masterton, 1983; Bortoff and Strick, 1993; Lemon and Griffiths, 2005). From a functional point of view, it appears that the CS system does not fully exploit its rich anatomical infrastructure, which could potentially generate multiple parallel loops of information processing.

\section{References}

Asher I, Zinger N, Yanai Y, Israel Z, Prut Y (2010) Population-based corticospinal interactions in macaques are correlated with visuomotor processing. Cereb Cortex 20:241-252. CrossRef Medline

Bennett KM, Lemon RN (1996) Corticomotoneuronal contribution to the fractionation of muscle activity during precision grip in the monkey. J Neurophysiol 75:1826-1842. Medline

Bortoff GA, Strick PL (1993) Corticospinal terminations in two new-world primates: further evidence that corticomotoneuronal connections provide part of the neural substrate for manual dexterity. J Neurosci 13:51055118. Medline

Boudrias MH, McPherson RL, Frost SB, Cheney PD (2010) Output properties and organization of the forelimb representation of motor areas on the lateral aspect of the hemisphere in rhesus macaques. Cereb Cortex 20: 169-186. CrossRef Medline

Brosch M, Bauer R, Eckhorn R (1997) Stimulus-dependent modulations of correlated high-frequency oscillations in cat visual cortex. Cereb Cortex 7:70-76. CrossRef Medline

Cheney PD, Fetz EE (1980) Functional classes of primate corticomotoneuronal cells and their relation to active force. J Neurophysiol 44:773-791. Medline

Cheney PD, Fetz EE (1985) Comparable patterns of muscle facilitation evoked by individual corticomotoneuronal $(\mathrm{CM})$ cells and by single intracortical microstimuli in primates: evidence for functional groups of CM cells. J Neurophysiol 53:786-804. Medline

Churchland MM, Cunningham JP, Kaufman MT, Ryu SI, Shenoy KV (2010) Cortical preparatory activity: representation of movement or first cog in a dynamical machine? Neuron 68:387-400. CrossRef Medline 
Dum RP, Strick PL (1991) The origin of corticospinal projections from the premotor areas in the frontal lobe. J Neurosci 11:667-689. Medline

Dum RP, Strick PL (1996) Spinal cord terminations of the medial wall motor areas in macaque monkeys. J Neurosci 16:6513-6525. Medline

Duque J, Labruna L, Verset S, Olivier E, Ivry RB (2012) Dissociating the role of prefrontal and premotor cortices in controlling inhibitory mechanisms during motor preparation. J Neurosci 32:806-816. CrossRef Medline

Fetz EE, Cheney PD (1980) Postspike facilitation of forelimb muscle activity by primate corticomotoneuronal cells. J Neurophysiol 44:751772. Medline

Fetz EE, Cheney PD (1987) Functional relations between primate motor cortex cells and muscles: fixed and flexible. Ciba Found Symp 132: 98-117. Medline

He SQ, Dum RP, Strick PL (1993) Topographic organization of corticospinal projections from the frontal lobe: motor areas on the lateral surface of the hemisphere. J Neurosci 13:952-980. Medline

Heffner RS, Masterton RB (1983) The role of the corticospinal tract in the evolution of human digital dexterity. Brain Behav Evol 23:165-183. CrossRef Medline

Humphrey DR, Gold R, Reed DJ (1984) Sizes, laminar and topographic origins of cortical projections to the major divisions of the red nucleus in the monkey. J Comp Neurol 225:75-94. CrossRef Medline

Kuypers HG, Lawrence DG (1967) Cortical projections to the red nucleus and the brain stem in the Rhesus monkey. Brain Res 4:151-188. CrossRef Medline

Kuypers HGJM (1973) The anatmomical organization of the descending pathways and their contribution to motor control especially in primates. In: New developments in EMG and clinical neurophysiology (Desmedt JE, ed), pp 38-68. Basel, Switzerland: Karger.

Lemon RN, Griffiths J (2005) Comparing the function of the corticospinal system in different species: organizational differences for motor specialization? Muscle Nerve 32:261-279. CrossRef Medline

Martino AM, Strick PL (1987) Corticospinal projections originate from the arcuate premotor area. Brain Res 404:307-312. CrossRef Medline

Moll L, Kuypers HG (1977) Premotor cortical ablations in monkeys: contralateral changes in visually guided reaching behavior. Science 198:317-319. CrossRef Medline

Monakow KH, Akert K, Künzle H (1979) Projections of precentral and premotor cortex to the red nucleus and other midbrain areas in Macaca fascicularis. Exp Brain Res 34:91-105. Medline

Murray EA, Coulter JD (1981) Organization of corticospinal neurons in the monkey. J Comp Neurol 195:339-365. CrossRef Medline

Nishimura Y, Onoe H, Morichika Y, Perfiliev S, Tsukada H, Isa T (2007) Time-dependent central compensatory mechanisms of finger dexterity after spinal cord injury. Science 318:1150-1155. CrossRef Medline
Perlmutter SI, Maier MA, Fetz EE (1998) Activity of spinal interneurons and their effects on forearm muscles during voluntary wrist movements in the monkey. J Neurophysiol 80:2475-2494. Medline

Porter R, Lemon RN (1993) Corticospinal function and voluntary movement. Oxford, UK: Clarendon.

Prut Y, Fetz EE (1999) Primate spinal interneurons show pre-movement instructed delay activity. Nature 401:590-594. CrossRef Medline

Prut Y, Perlmutter SI (2003a) Firing properties of spinal interneurons during voluntary movement. I. State-dependent regularity of firing. J Neurosci 23:9600-9610. Medline

Prut Y, Perlmutter SI (2003b) Firing properties of spinal interneurons during voluntary movement. II. Interactions between spinal neurons. J Neurosci 23:9611-9619. Medline

Ralston DD, Ralston HJ 3rd (1985) The terminations of corticospinal tract axons in the macaque monkey. J Comp Neurol 242:325-337. CrossRef Medline

Schieber MH (2002) Motor cortex and the distributed anatomy of finger movements. Adv Exp Med Biol 508:411-416. CrossRef Medline

Shalit U, Zinger N, Joshua M, Prut Y (2012) Descending systems translate transient cortical commands into a sustained muscle activation signal. Cereb Cortex 22:1904-1914. CrossRef Medline

Shimazu H, Maier MA, Cerri G, Kirkwood PA, Lemon RN (2004) Macaque ventral premotor cortex exerts powerful facilitation of motor cortex outputs to upper limb motoneurons. J Neurosci 24:1200-1211. CrossRef Medline

Shinoda Y, Yokota J, Futami T (1981) Divergent projection of individual corticospinal axons to motoneurons of multiple muscles in the monkey. Neurosci Lett 23:7-12. CrossRef Medline

Takei T, Seki K (2010) Spinal interneurons facilitate coactivation of hand muscles during a precision grip task in monkeys. J Neurosci 30:17041-17050. CrossRef Medline

Valero-Cuevas FJ, Venkadesan M, Todorov E (2009) Structured variability of muscle activations supports the minimal intervention principle of motor control. J Neurophysiol 102:59-68. CrossRef Medline

Wise SP, Weinrich M, Mauritz KH (1986) Movement-related activity in the premotor cortex of rhesus macaques. Prog Brain Res 64:117-131. CrossRef Medline

Yanai Y, Adamit N, Harel R, Israel Z, Prut Y (2007) Connected corticospinal sites show enhanced tuning similarity at the onset of voluntary action. J Neurosci 27:12349-12357. CrossRef Medline

Yanai Y, Adamit N, Israel Z, Harel R, Prut Y (2008) Coordinate transformation is first completed downstream of primary motor cortex. J Neurosci 28:1728-1732. CrossRef Medline 